\title{
Non-symbiotic hemoglobin and its relation with hypoxic stress
}

\author{
Alejandro Riquelme $^{1^{*}}$, and Patricio Hinrichsen ${ }^{2}$
}

Today we know that several types of hemoglobins exist in plants. The symbiotic hemoglobins were discovered in 1939 and are only found in nodules of plants capable of symbiotically fixing atmospheric N. Another class, called non-symbiotic hemoglobin, was discovered $32 \mathrm{yr}$ ago and is now thought to exist throughout the plant kingdom, being expressed in different organs and tissues. Recently the existence of another type of hemoglobin, called truncated hemoglobin, was demonstrated in plants. Although the presence of hemoglobins is widespread in the plant kingdom, their role has not yet been fully elucidated. This review discusses recent findings regarding the role of plant hemoglobins, with special emphasis on their relationship to plants adaptation to hypoxia. It also discusses the role of nitric oxide in plant cells under hypoxic conditions, since one of the functions of hemoglobin appears to be modulating nitric oxide levels in the cells.

Key words: Anaerobic, hemoglobin, hypoxia, nitric oxide, oxygen transport.

\section{INTRODUCTION}

Hemoglobins are found ubiquitously in eukaryotes and in many bacteria (Wittenberg and Wittenberg, 1990; Bhattacharya et al., 2013). Three types of hemoglobins have been described in plants, which have been classified as symbiotic, non-symbiotic and truncated. Symbiotic hemoglobins are the best known; they are found mainly in the nodules of plants with symbiotic $\mathrm{N}$ fixation. In this case their function is to regulate the supply of oxygen to the $\mathrm{N}$-fixing bacteria (Appleby, 1992; Ott et al., 2005). Nonsymbiotic hemoglobins (nsHb), as their name indicates, are not involved in symbiotic $\mathrm{N}$ fixation; apparently this type of protein exists in the entire vegetable kingdom, including plants with symbiotic capacity. Expression has been found in seeds, roots and other organs, both in monocotyledons and dicotyledons (Hill, 1998; Thiel et al., 2011). The ubiquity of the nsHb, along with the fact that they are probably evolutionary predecessors of the symbiotic hemoglobins, suggests that they have an important role in plant metabolism (Hebelstrup et al., 2006). The most recently discovered hemoglobins, the truncated hemoglobins (trHb), are also thought that exist throughout the entire plant kingdom; they share some characteristics with the $\mathrm{nsHb}$ but they are smaller in size

${ }^{1}$ Centro de Estudios Avanzados en Fruticultura (CEAF), Camino Las Parcelas 882, sector Los Choapinos, Rengo, Chile.

*Corresponding author (alriquel@gmail.com).

${ }^{2}$ Instituto de Investigaciones Agropecuarias, INIA La Platina, Santa Rosa 11610, La Pintana, Santiago, Chile.

Received: 5 December 2014.

Accepted: 4 May 2015.

doi:10.4067/S0718-58392015000300009
(Watts et al., 2001; Wittenberg et al., 2002; Kumar et al., 2013). However, their different origin, biochemical characteristics and tertiary structure suggest that trHb have cellular functions different from those of nsHb.

In spite of the extensive presence of plant hemoglobins in the plant kingdom, their functions are not yet clear. The objective of this review is discussing recent findings regarding the role of plant hemoglobins, with special emphasis on their relationship to adaptation of the plants to the hypoxia. It also discusses their function in modulating nitric oxide levels in the cells.

\section{Historical overview of plant hemoglobins}

In 1939 Kubo was the first to describe a plant hemoglobin, from the Rhizobium nodules in roots of a soybean plant (Kubo, 1939). The isolation of hemoglobin from Parasponia andersonii (Planch.) Planch, which is not a legume, demonstrated that the presence of hemoglobin was not restricted to legumes (Appleby et al., 1983). This discovery was remarkable not only for being the first hemoglobin found in a non-legume, but also because it was expressed in different parts of the plant outside of the nodule. Later, the same research group demonstrated hemoglobin expression in Trema tomentosa (Roxb.) H. Hara, showing for the first time its presence in a nonnodulating species (Bogusz et al., 1988). The discovery of hemoglobin in barley and the demonstration that this hemoglobin is also present in other cereals such as maize, wheat and rye led to a series of reports on hemoglobins in other species (Taylor et al., 1994). In 1994, two hemoglobins were found in rice (Sasaki et al., 1994) and one in chloroplasts of the alga Chlamydomonas eugametos (Couture et al., 1994). At the beginning of the $21^{\text {st }}$ century, 
another type of hemoglobin was found in Arabidopsis with structural similarities to bacteria, protozoan, and algae hemoglobin (Watts et al., 2001; Wittenberg et al., 2002); it is therefore known as truncated hemoglobin. To date more than 50 plant hemoglobins have been found in over 20 species (Garrocho-Villegas et al., 2007; BustosSanmamed et al., 2011; Bhattacharya et al., 2013).

\section{Evolution of plant hemoglobins}

Hemoglobins are widely distributed in the Kingdom Plantae; they have been detected in primitive plants such as the bryophyte Physcomitrella patens as well as in monocots and dicots. It is believed that plant and animal hemoglobins derive from the same ancestral globin, about 1.5 billion years ago, and have evolved by vertical evolution (Zhu and Riggs, 1992). The monomeric units of animal and plant hemoglobins fold into a highly conserved globin-type structure. Both symbiotic and non-symbiotic hemoglobins have been found in dicotyledons (Trevaskis et al., 1997), while only non-symbiotic hemoglobins have been detected in monocotyledons (Figure 1) (Hunt et al., 2001; Garrocho-Villegas et al., 2007).

It has been reported that genes of symbiotic and nonsymbiotic hemoglobins have three introns and four exons (Arredondo-Peter et al., 1998). The distribution of introns in the genes of all known hemoglobins was found to be the same as the one detected in the symbiotic hemoglobins of soybean and Parasponia (Dordas et al., 2003; Gupta et al., 2011), suggesting that this pattern has been maintained since the ancestral development of hemoglobins. NsHbs are widely distributed in the plant kingdom while symbiotic hemoglobins are restricted to the nodules of $\mathrm{N}$-fixing plants, which suggests that the latter arose from a duplication of a hemoglobin gene in which one of the copies was sequestered to perform a specific function in the nodules (Appleby, 1992). Since then the two types

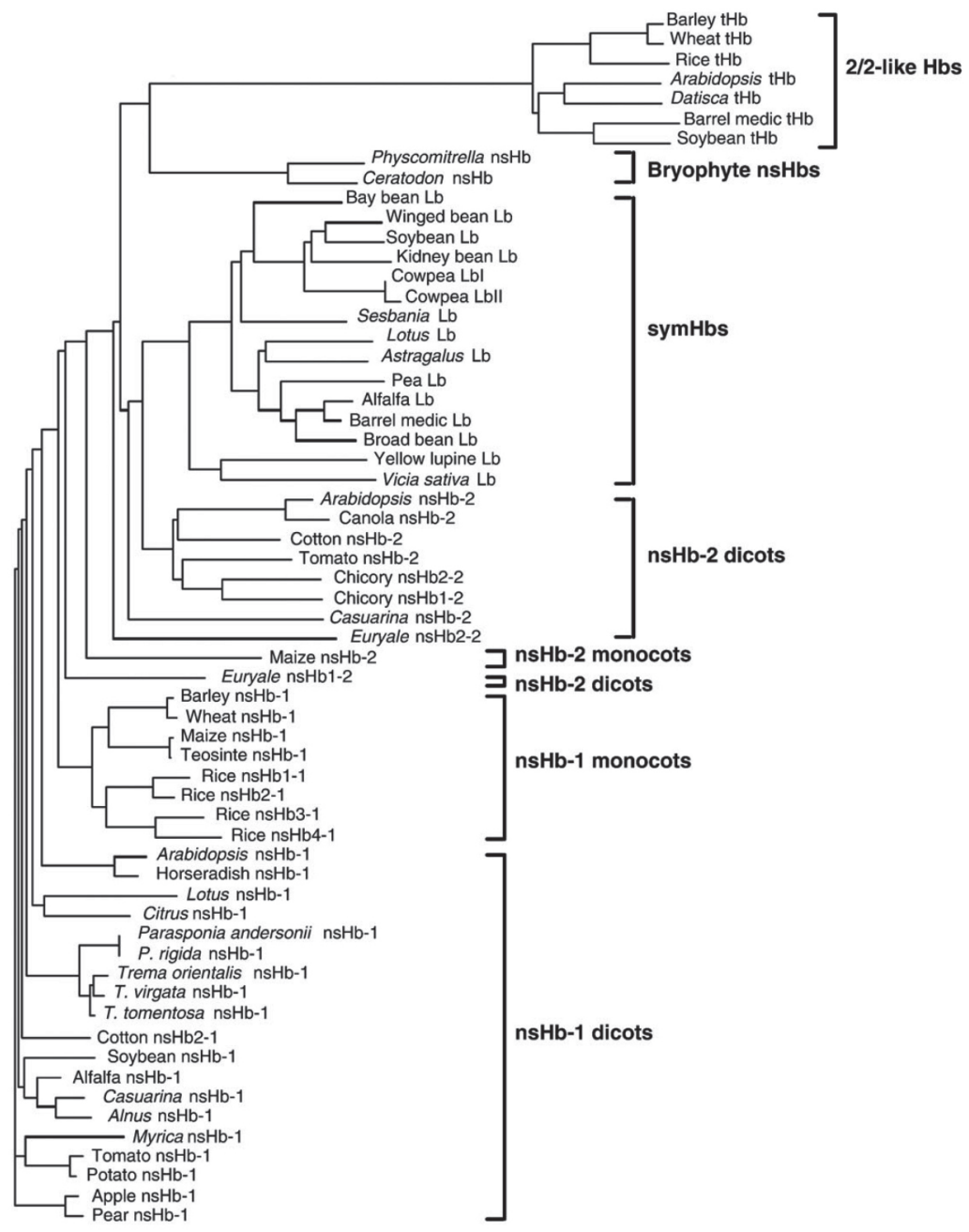

Source: Garrocho-Villegas et al. (2007) and Thompson et al. (1997).

Figure 1. Cluster (phenetic) analysis of selected plant hemoglobins (Hbs). Hb sequences were aligned and the phenogram was constructed using the Clustal X program (Conway Institute, University College Dublin, Dublin, Ireland). 
of plant hemoglobins have evolved independently with different functions. It has been reported that plants have differential expression of at least three genes that code for non-symbiotic hemoglobins with potentially distinct biochemical properties (Arredondo-Peter et al., 1998). For example, in Arabidopsis hemoglobin AtnsHb1 has the typical sequences and oxygen-binding characteristics of $\mathrm{NsHb}$ proteins, while AtnsHb2 has greater similarity with the sequences and oxygen-binding characteristics of symbiotic hemoglobins (Arredondo-Peter et al., 1998). The expression pattern of these three genes is different; AtnsHb1 is induced by hypoxia and nitrate, while AtnsHb2 is induced by cold stress and AtnsHb3 is expressed in roots and shots and is repressed by hypoxia (Trevaskis et al., 1997; Wang et al., 2000; Watts et al., 2001).

\section{Structure of non-symbiotic hemoglobins}

The structure of nsHb is similar to that of hemoglobins of many organisms, since all have the globin-type folding that consists of seven or eight alpha helices identified with the letters A to $\mathrm{H}$ (Figure 2A). The cleft for the heme group is a conserved region formed by helices $\mathrm{E}$ and $\mathrm{F}$ (Hoy and Hargrove, 2008; Spyrakis et al., 2011a) (Figure 2B). It is in this region that the distal (E7) and proximal (E8) histidines coordinate $\mathrm{Fe}$ of the heme group. The $\mathrm{Fe}$ atom is also coordinated by the four-pyrrole rings of the heme group (Figure 2B). The nsHbs of plants were initially divided into two subgroups, called nsHb1 and nsHb2 (Trevaskis et al., 1997; Hunt et al., 2001), based on phylogenetic analyses, patterns of gene expression and the oxygen binding properties of these proteins. The first crystallized hemoglobin in a monocot was the rice nsHb1 (nsHb1-rice) (Hargrove et al., 2000). Its crystalline structure showed a characteristic hexacoordination, with the distal histidine bound to the heme group $\mathrm{Fe}$ and a conserved phenylalanine residue (Phe-B10) in the B helix that interacts with the distal histidine (Hargrove et al., 2000; Smagghe et al., 2006; Spyrakis et al., 2011a). However, there are also differences between the nsHb1 proteins of different species; for example, tertiary structures of nsHb1-rice and nsHb1-barley are different (Bykova et al., 2006; Hoy and Hargrove, 2008; Spyrakis et al., 2011b). In maize, a computer model predicted a structure similar to that of nsHb1-rice (Saenz-Rivera et al., 2004); however, NsHb1-maize has more inter-helix links than nsHb1-rice, which suggests reduced flexibility of nsHb1-maize and a possible kinetic difference in its bond with the ligand (Smagghe et al., 2006).

There is not much information about the structures of dicotyledon nsHbs. The nsHb of tomato has hexacoordination of the $\mathrm{Fe}^{+3}$ ion and a mixture of pentacoordination and hexacoordination of the $\mathrm{Fe}^{+2}$ ion (Ioanitescu et al., 2005). The AtHb1 of Arabidopsis has a $40 \%$ pentacoordination (Bruno et al., 2007; Spyrakis et al., 2011a). The structure and functioning of $\mathrm{NsHb} 2$ has been described in detail only for Arabidopsis (Trevaskis et al., 1997; Bruno et al., 2007), in which AtHb2 has a smaller and more confined cleft than AtNsHb1 (Spyrakis et al., 2011b).

NsHb1 forms structural dimers composed of two identical subunits, each with a hexacoordinated hemeFe complex (Figure 2A). NsHb1 has a higher rate of Fe reduction, which is probably mediated by a cysteine residue. This cysteine may form a reversible covalent bond between two monomers as indicated by mass spectrometry, which in addition to its structural role would impede self-oxidation of the molecule (Igamberdiev et al., 2011; Gupta et al., 2011).

\section{Biochemistry of the oxygen-hemoglobin bond}

The velocity of oxygen binding and liberation depends on the type of hemoglobin and is an essential characteristic of its cell function. For example, leghemoglobins (symbiotic) have an oxygen affinity 20 fold higher than
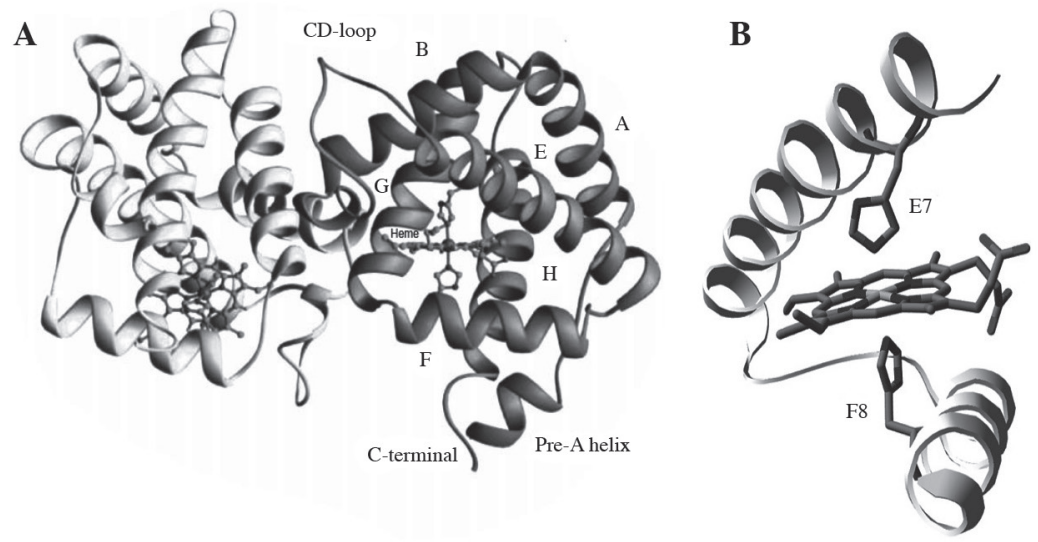

A: Garrocho-Villegas et al. (2007); B: Hoy and Hargrove (2008).

Figure 2. Crystalline structure of rice non-symbiotic hemoglobin (nsHb1). (A) Dimeric structure and alpha helices A-H. (B) Coordination zone of rice nsHb1. Shown are the $\mathrm{E}$ and $\mathrm{F}$ helices, the distal histidine (E7), the proximal histidine (F8) and the heme group. 
myoglobin. The kinetics of association of $\mathrm{O}_{2}$ with $\mathrm{nsHb}$ $\left(68 \mu \mathrm{mol}^{-1} \mathrm{~s}^{-1}\right)$ is four fold higher than in myoglobin and one order of magnitude less than that of leghemoglobin (Duff et al., 1997). It is known that all hemoglobins reversibly bind to $\mathrm{O}_{2}$ due to possessing a heme group that has a molecule of Fe. The heme group of $\mathrm{nsHb}$ is hexacoordinated and interacts with the distal histidine; it has high oxygen affinity and a low disassociation constant (Igamberdiev et al., 2011). This low disassociation constant is due to the rapid binding of the oxygen, which produces conformational changes in the protein and, as a consequence, a slow disassociation of the oxygen from the heme group. The equilibrium disassociation constant of rice is $0.038 \mathrm{~s}^{-1}$ (Kundu et al., 2003). This low disassociation constant indicates that this hemoglobin remains oxygenated under very low oxygen pressure, which makes it improbable that nsHb serve as transporters, storers, or sensors of oxygen (Hill, 1998).

\section{Kinetics of the bond with the ligand}

As with other globins, plant hemoglobins may bind to several different ligands, such as $\mathrm{O}_{2}, \mathrm{CO}, \mathrm{NO}$, and $\mathrm{CN}$. The affinity constants of nsHb for $\mathrm{O}_{2}$ and $\mathrm{CO}$ and their rate of binding $\mathrm{NO}$ are approximately an order of magnitude lower than the respective values of the ferric forms of bacterial hemoglobins. The nsHb have similar values to the $\mathrm{O}_{2}$-binding constants of bacterial globins $\left(38 \mu \mathrm{M}^{-1} \mathrm{~s}^{-1}\right.$ for $E$. coli and $68 \mu \mathrm{M}^{-1} \mathrm{~s}^{-1}$ for rice), but have lower $\mathrm{O}_{2}$ liberation constants $\left(0.44 \mathrm{~s}^{-1}\right.$ for $E$. coli and $0.038 \mathrm{~s}^{-1}$ for rice), which results in higher affinity constants (Gardner et al., 1998; Farrés et al., 2005; Smagghe et al., 2008). The oxygen binding kinetics are different for the two types of nsHb; nsHb1 has an unusually high affinity for $\mathrm{O}_{2}$ (Arredondo-Peter et al., 1997; Duff et al., 1997; Trevaskis et al., 1997), much higher than that found for $\mathrm{nsHb} 2$, which implies they have different functions in the cell (Trevaskis et al., 1997). Also, nsHb1 has a very low equilibrium disassociation constant, less than that of cytochrome oxidase, which suggests that it could not supply $\mathrm{O}_{2}$ to the terminal oxidase of the mitochondria. The low disassociation constant of nsHb1 indicates that it would not be capable of facilitating $\mathrm{O}_{2}$ diffusion. Thus it seems improbable that nsHb1 functions as an oxygen transporter (Trevaskis et al., 1997; Arredondo-Peter et al., 1997) or sensor (Duff et al., 1997). Experiments on the expression of different nsHbs in transgenic plants have shown that the level of total adenylates is maintained constant when these plants are exposed to low oxygen concentrations, while in the untransformed (or control) plant lines the energy level is reduced by about $35 \%$. This suggests that the function of nsHbs in plants is to maintain the energetic state of the cell in low oxygen environments (Sowa et al., 1998; Igamberdiev et al., 2011).

\section{Factors affecting nsHb expression}

The production of the two classes of $\mathrm{nsHb}$ is organ-specific, and expressed as a response to a variety of stimuli. The $\mathrm{nsHb}$ are expressed in calli, cell suspensions, seeds, roots, and stems of monocotolyledons and dicotolyledons (Hill, 1998). They are generally present in low concentrations (1$20 \mathrm{nmol} \mathrm{g}^{-1}$ fresh weight) in these tissues and organs (Duff et al., 1997). Hemoglobins are expressed in plant tissue in response to specific metabolic stresses. Thus, hemoglobin is induced by hypoxia in barley aleurone layers, maize roots and embryos (Taylor et al., 1994). In addition to their induction by hypoxic stress (Taylor et al., 1994), they are also found in rapidly growing tissues such as root tips of germinating seeds (Hill, 1998). Elevated sucrose levels have also been shown to increase hemoglobin gene expression in Arabidopsis (Trevaskis et al., 1997).

\section{Regulation by $\mathrm{O}_{2}$ and ATP levels}

Hemoglobins are expressed in response to different types of metabolic stress. The AtnsHb1s, which are active during germination, may be induced by hypoxia and by a high level of sucrose (Hunt et al., 2001). In barley, these proteins are expressed in the aleurone layers of the seed and in roots under low oxygen stress (Taylor et al., 1994). Inhibitors of the respiratory chain such as cyanide, oligomycin and dinitrophenol, which inhibit ATP production, also induce nsHblexpression, which suggests that their expression is not directly influenced by $\mathrm{O}_{2}$ levels but rather by the energy levels or indirectly by the action of ATP (Nie and Hill, 1997). It has also been observed that nsHb1 genes are generally induced in tissues with rapid growth such as root meristems of germinated seeds (Hill, 1998; Igamberdiev et al., 2011). Again, this fact indicates that the presence of this protein in rapidly growing tissues during germination may be due to hypoxic conditions (Guy et al., 2002; Hebelstrup et al., 2006). The results of Bogusz et al. (1990) support this idea; using promoters of Parasponia and Trema with the GUS reporter gene they showed that the promoter regions of $\mathrm{nsHb}$ genes direct expression mainly to root tips and the vascular bundle of transformed tobacco plants and to the roots of transformed Lotus japonicus.

\section{Regulation by osmotic stress and minerals}

It has been reported that nsHb1 genes are induced by osmotic stress and high salinity (Taylor et al., 1994; Trevaskis et al., 1997; Lira-Ruan et al., 2001; Zhao et al., 2008; Almada et al., 2013), by treatments with nitrate, nitrite, nitric oxide, salicylic acid, methyl jasmonic acid, and $\mathrm{H}_{2} \mathrm{O}_{2}$ (Wang et al., 2000; Sakamoto et al., 2004; Ohwaki et al., 2005; Qu et al., 2006; Sasakura et al., 2006), as well as by deficiencies in minerals such as P, K, and Fe (Wang et al., 2003). ZmHb mRNA levels in maize seedlings are induced by high-salt and osmotic stresses in addition to hypoxic stress (Zhao et al., 2008). In tomato, after $1 \mathrm{~h}$ of $\mathrm{P}$ deprivation, nsHb1 mRNA transcript levels had increased relative to the control, and peaked at $12 \mathrm{~h}$ after removal of this nutrient from the solution. 
Both $\mathrm{K}$ and Fe deficiencies increased the nsHb1 mRNA abundance within $3 \mathrm{~h}$ of nutrient deprivation (Wang et al., 2003). Hemoglobin is also induced by nitrate ions in aleurone layers and in Arabidopsis roots (Nie and Hill, 1997; Wang et al., 2000). Two nsHb1 genes, ORYsa GLB1a and ORYsa GLB1b, were strongly induced by nitrate, nitrite, and nitric oxide (Ohwaki et al., 2005). The induction of the $\mathrm{Hb}$ gene by hypoxia is linked to a decrease in ATP levels and is mediated by $\mathrm{Ca}^{2+}$ (Hebelstrup et al., 2007). This background indicates that expression of $\mathrm{nsHb}$ at transcript levels is regulated under multiple stress conditions, suggesting an important role of $\mathrm{nsHb}$ in plant stress tolerance.

\section{Hormonal regulation}

The effects of hormones on the expression of hemoglobins have been studied. NsHb1 is induced by 1-aminocyclopropane-1-carboxylic acid (ACC) and cytokinins (CKs), while nsHb2 is repressed by these hormones (Igamberdiev et al., 2005; Bustos-Sanmamed et al., 2011). Hormones may also have opposing effects depending on the plant organ; for example, CKs produce strong induction of nsHb1 in roots of Lotus japonicus and negative regulation in the nodules. Polyamines and jasmonic acid cause strong induction of nsHb1 in roots. These effects may be mediated by NO (Igamberdiev et al., 2005). When the expression of nsHb was suppressed in maize cells an elevated formation of ethylene was observed (Manac'h-Little et al., 2005), which indicates a potential function of $\mathrm{nsHb}$ in the regulation of ethylene levels. The levels of ethylene and NO also increased when the expression of nsHb was suppressed, suggesting that NO has a promoting effect on ethylene biosynthesis (Igamberdiev et al., 2005).

Characterization of the transcription factors of Oryza sativa $\mathrm{L}$. that regulate the expression of the $\mathrm{nsHb}$ genes indicated that cytokinins induce or activate $O_{s N S H B 2}$, mediated by ARR1 (Arapidopsis Response Regulator 1), a factor which contains a Myb-like DNA binding domain (Ross et al., 2004), while the expression of HsHb1 in tissues under hypoxic stress depends on an incomplete transcription factor, Mybleu, which is incapable of binding to DNA (Mattana et al., 2007).

\footnotetext{
Physiological functions of non-symbiotic hemoglobins Although the functions of the nsHb have not been clearly established, their presence in the entire plant kingdom and their expression in metabolically active tissues suggest that they play an important role in plant metabolism. Since they were discovered, diverse biochemical and physiological functions have been attributed to plant hemoglobins, including the transport, storage, and sensing of oxygen. The nsHb have also been associated with the transport of ligands such as $\mathrm{CO}, \mathrm{NO}$, and fatty acids, as well as the function of an oxygen scavenger (ArredondoPeter et al., 1998; Jokipii-Lukkari et al., 2009; Thiel et
}

al., 2011). Knock-out silencing studies in A. thaliana have demonstrated the fundamental role of nsHb during plant development, showing that at least one functional $\mathrm{nsHb}$ gene is essential for plant survival (Hebelstrup et al., 2006; Dordas, 2009). The information above shows that the presence of hemoglobins is vitally important for plant development and also in the stress tolerance response to hypoxia. Considering the importance of the latter aspect, we will discuss the role that the $\mathrm{nsHb}$ may fulfill during hypoxia stress.

\section{Function of nsHb in stress due to hypoxia and its interaction with nitric oxide}

Although the function of the nsHbs is unknown, analysis of their gene expression is a useful tool to approach the function of these proteins. It has been shown that expression of $\mathrm{nsHb}$ genes is modified when plants grow under different stress conditions, which suggests that the function of the nsHb genes is related to a plant's response to these stress conditions (Hebelstrup et al., 2006; Sairam et al., 2009; Almada et al., 2013). The AtnsHb1 (At21660) gene is expressed in roots of plants that grow under normal conditions, and is overexpressed in leaves and roots when plants are under hypoxic conditions (Hebelstrup et al., 2006; Dordas, 2009). The constitutive expression of barley nsHb1 in maize cell lines maintained normal cell levels of adenine nucleotides (ATP, ADP, and AMP) and the energy charge under conditions of hypoxia, while cells in which hemoglobin expression was suppressed had lower levels of these parameters (Igamberdiev et al., 2011). Similarly, in transformed alfalfa that constitutively expressed barley nsHb1, roots grew normally under hypoxia, while roots of the wild type and of plants in which nsHb1 expression was suppressed grew slowly (Dordas et al., 2003). It is improbable that these effects are produced by nsHb1 acting as a signal which triggers the activation of other genes, due to the low disassociation constant of the oxyhemoglobin complex (Table 1) (Duff et al., 1997). For this reason it has been suggested that nsHb1 helps to maintain the energy state of cells in environments with low oxygen and acts by promoting glucolytic flux via NADH oxidation, which results in an increase in phosphorylation at the substrate level (Dordas, 2009). Thus, nsHb1 could be used to sequester oxygen in hypoxic environments, and would provide an oxygen source to oxidate NADH and in this way obtain ATP for cell growth and development.

In bacteria and yeasts, NO may be eliminated by flavohemoglobin through its dioxygenase activity (Gardner et al., 1998). Dordas et al. (2003; 2004) proposed that in plants at least one function of hemoglobin induced by hypoxic stress is to modulate the NO level in the cell. Using electronic paramagnetic resonance (EPR), these researchers demonstrated the presence of hemeNO complexes in maize cell cultures and in cultures of alfalfa roots under hypoxic conditions. They also 
Table 1. Rate and affinity constants for the binding of oxygen to myoglobin and nonsymbiotic hemoglobin and leghemoglobin.

\begin{tabular}{|c|c|c|c|}
\hline Type & $\begin{array}{c}\text { Oxygen association } \\
\text { constant }\left(k_{\text {on }}\right)\end{array}$ & $\begin{array}{l}\text { Oxygen dissociation } \\
\text { constant }\left(k_{\text {off }}\right)\end{array}$ & $\begin{array}{l}\text { Affinity constant } \\
(K)\end{array}$ \\
\hline & $\mu \mathrm{M}^{-1} \mathrm{~s}^{-1}$ & $\mathrm{~s}^{-1}$ & $\mu \mathrm{M}^{-1}$ \\
\hline Sperm whale myoglobin ${ }^{a}$ & 17 & 15 & 1.1 \\
\hline Soybean leghemoglobin ${ }^{\mathrm{b}}$ & 130 & 5.6 & 23 \\
\hline Rice class 1 non-symbiotic hemoglobin & 68 & 0.038 & $200^{\mathrm{b}}$ \\
\hline
\end{tabular}

$\mathrm{k}_{\mathrm{on}}$ : Association constant (affinity with which $\mathrm{O}_{2}$ binds to $\left.\mathrm{Fe}^{2+}\right) ; \mathrm{k}_{\text {off }}$ : dissociation constant $\left(\mathrm{Fe}^{2+}\right.$ affinity for the released oxygen); $\mathrm{K}$ : affinity constant $\left(\mathrm{k}_{\mathrm{on}} / \mathrm{k}_{\mathrm{off}}\right)$. aData from Harorove et al. (2000).

${ }^{b}$ Data from Kundu et al. (2003).

showed that there is no EPR signal for the complex in aerobic systems, although hemoglobin is present. Using NO trapping it has been demonstrated that maize cells form significant quantities of NO during the first $24 \mathrm{~h}$ of hypoxia treatment. Perazzolli et al. (2004) found that AtnsHb1 neutralized the production of $\mathrm{NO}$ by forming S-nitroso-hemoglobin and this way reduced the emission of NO during hypoxic stress, reaffirming its possible role in the detoxification of NO.

Hemoglobins have been implicated in $\mathrm{NAD}^{+}$ regeneration, which is necessary in order to maintain glycolysis under conditions of hypoxia (Figure 3) (Hill, 1998). This is based on observations that the activity of alcohol dehydrogenase and $\mathrm{CO}_{2}$ production decrease under hypoxic conditions in maize cells that constitutively express barley hemoglobin (Sowa et al., 1998). It has also been demonstrated that nitrate ion may act as final electron acceptors in the denitrification process in inundated soils, which suggests that it may play a similar role in plants (Crawford, 1978). Although we still do not know the mechanism of NO formation under hypoxic conditions, there are results which support the pathway involving the enzyme nitrate reductase (Figure 3) (Yamasaki and Sakihama, 2000; Stöhr et al., 2001; Gupta et al., 2011; Sairam et al., 2012), which consumes two moles of NADH for each mole of NO formed. Previous data were already showed that this enzyme is activated when roots are subjected to hypoxia (Botrel and Kaiser, 1997). The NO formed rapidly reacts with oxyhemoglobin, generating nitrate and metahemoglobin [nsHb $\left.\left(\mathrm{Fe}^{+3}\right)\right]$ (Figure 3) (Doyle and Hoekstra, 1981; Igamberdiev and Hill, 2004). This metabolic pathway of $\mathrm{NO}$, in which nitrate is recycled, would be advantageous for plant cells exposed to prolonged conditions of soil flooding when the content of nitrates is very reduced (Tiedje et al., 1982; Burgin and Hamilton, 2007). This sequence of events is known as the $n \mathrm{sHb} / \mathrm{NO}$ cycle (Figure 3). In this cycle, nsHb- $\mathrm{Fe}^{+3}$ may be reduced to $\mathrm{nsHb}-\mathrm{Fe}^{+2}$ by a NADH-dependent reductase (Jakob et al., 1992; Poole and Hughes, 2000). The NADH-dependent reductase of plant cells under hypoxia would supply the $\mathrm{NAD}^{+}$needed to keep glycolysis functioning (Figure 3 ). The overall system oxidizes 2.5 moles of NADH for each mole of nitrate recycled during the reaction, which maintains the redox state and energy during hypoxia, resulting in reduced production of ethanol and lactic acid. Thus, the nsHb/NO cycle would play an important role, being an alternative to the classical fermentation pathways, and also collaborating to retard the onset of cell death. In primary roots this pathway may provide enough time for the plant to develop adventitious roots, which are necessary to prolong survival under hypoxic conditions (Igamberdiev et al., 2005).

The hexacoordinated nsHbs are generally known for their high sequence identity between species, which suggests that they are proteins with key biological functions at the cellular level. However, although there are various hypotheses, their physiological functions are not precisely known. These hypotheses include: (i) the $\mathrm{nsHb}$ may act as sensors of the $\mathrm{O}_{2}$ concentration in plant cells, so when the $\mathrm{O}_{2}$ concentration decreases in the cell, this acts as a signal to initiate the anaerobic response; (ii) the $\mathrm{nsHb}$ may participate in the maintaining the energy level of the cells, oxidizing NADH and generating the $\mathrm{NAD}^{+}$necessary to maintain glucolysis and ATP levels; (iii) the nsHb may detoxify the cells of toxic compounds such as nitric oxide and carbon monoxide; (iv) the $\mathrm{nsHb}$ may have a function in the signal transduction pathways of phytohormones such as auxins, ethylene, jasmonic acid, salicylic acid, abscisic acid, and cytokinins. Research at the biochemical, biophysical, structural and functional levels is necessary to fully understand the function of these proteins in plant cells.

Although there is convincing evidence that nsHb1 participates in the detoxification of NO, some of the essential questions about its functioning and properties are still unanswered. In plant cells the presence of NO has been evidenced in the mitochondria, cytosol, perixosomes, and apoplasts (Blokhina and Fagerstedt, 2010), while the nsHb1 proteins are apparently located in the nucleus and cytosol (Serégelyes et al., 2000) but absent from mitochondria and peroxisomes (Hebelstrup et al., 2008). This inevitably raises questions about the role of nsHb1 in cellular energy.

According to the previously presented data, $\mathrm{nsHb}$ proteins may play a preponderant role in biotechnological perspective programs to improve crop production, under both normal and stressful conditions. They are thought to play an important role particularly in aiding plant responses to hypoxia. Holmberg et al. (1997) generated transgenic tobacco plants that constitutively synthesized the hemoglobin 


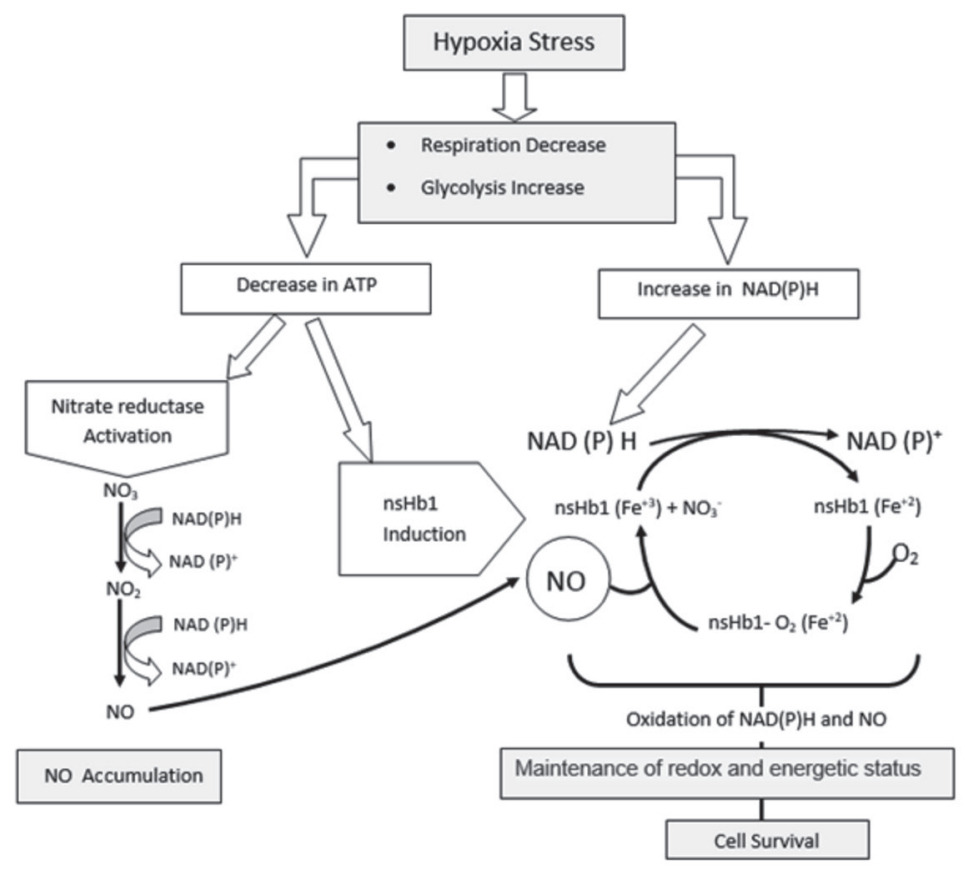

Modified from Dordas (2009).

Figure 3. Generalized scheme of the potential pathways of acclimation associated with non-symbiotic hemoglobin (nsHb1) and NO in an environment with low oxygen content. The beginning of hypoxia leads to a decrease in mitochondrial respiration, which produces an increase in NADH and a decrease in the level of ATP. Hypoxia induces the expression of the nsHb1 gene and the accumulation in nitrate, which is converted to NO. A reaction between NO and the oxygenated form of newly synthesized nsHb1 leads to oxidation and oxygenation of NO, resulting in restoration of the redox state and energy.

of Vitreoscilla bacteria ( $\mathrm{VHb}$ ), and proved that the transgenic plants increased their productivity compared to non-transformed plants. The two transformed lines showed higher growth rates and alterations in some metabolic pathways. Plants transgenic for $\mathrm{VHb}$ germinated 3-4 d before the non-transformed control plants and developed faster, accumulating 80\%-100\% more fresh weight after $35 \mathrm{~d}$. Li et al. (2005) obtained similar results with the overexpression of $\mathrm{VHb}$ in cabbage (Brassica oleracea L. var. capitata L.), where it also increased their tolerance to immersion stress. The transgenic cabbage plants with VHb grew normally during a prolonged period of immersion, while wild type plants died by the end of the treatment ( $\mathrm{Li}$ et al., 2005). The overexpression of nsHb1 also protected plants of Arabidopsis thaliana against severe effects of hypoxia, while plants that overexpressed a mutated nsHb1 with low oxygen affinity were just as susceptible to hypoxia as wild type plants ( $\mathrm{Li}$ et al., 2005). This suggests that the protection against hypoxia depends on the capacity of the hemoglobin to bind ligands such as oxygen with high affinity. The constitutive expression of $n s \mathrm{Hb} 1$ produced a reduction in the number of root hairs and an increase in the number of lateral roots (Hunt et al., 2002). The ectopic expression of nsHb1maize in transgenic tobacco improved the tolerance of plants to multiple stresses (inundation, salt, and osmotic), indicating an important role of nsHb1-maize in this tolerance (Zhao et al., 2008).

\section{CONCLUSIONS}

Research on non-symbiotic plant hemoglobins is still in a very early stage, although in recent years an appreciable amount of information has accumulated. Non symbiotic hemoglobins are broadly present across evolution; however, the function of these proteins is unknown. Non symbiotic hemoglobins are likely ancestors of an early form of hemoglobin that sequestered oxygen in low oxygen environments. It was suggested that non symbiotic hemoglobins act in plants to maintain energy status of cells in low oxygen environments and that they accomplish this effect by promoting glycolytic flux through NADH oxidation, resulting in increased substrate-level phosphorylation. In this way, hemoglobins providing a source of oxygen to oxidize NADH to provide ATP for cell growth and development. This in turn suggests that cells containing increased levels of hemoglobins will survive longer under low oxygen tension or high energy demand. Plant roots that express sufficient hemoglobin soon after exposure to hypoxic stress may modulate levels of nitric oxide, produced as a 
result of the stress, either through reaction of nitric oxide with oxyhemoglobin or through formation of nitrosylhemoglobin. Non symbiotic hemoglobin and nitric oxide are an alternative to fermentation to maintain lower redox potential. Hemoglobin may be pivotal in the short-term survival of plant root cells by regulating the levels of nitric oxide. This would prevent the accumulation of toxic ethanol and lactic acid, maintenance of ATP levels and energy charge, and delay the onset of cell death. In primary roots, this may provide sufficient time for the plant to develop adventitious roots and/or aerenchyma, needed for prolonged survival under hypoxia. The information included in this review suggests that the constitutive expression of the non-symbiotic hemoglobin gene in transgenic plants improves the survival of plants under hypoxic conditions and in other stresses. It remains to be verified if the increased productivity reported in annual species can be reproduced in perennial plant species such as fruit trees, allowing them to confront conditions of low oxygen content in the rhizosphere.

\section{ACKNOWLEDGEMENTS}

This work was supported by grants from CONICYT Regional/CEAF/R08I1001.

\section{LITERATURE CITED}

Almada, R., M.J. Arismendi, P. Pimentel, P. Rojas, P. Hinrichsen, M. Pinto, et al. 2013. Class 1 non-symbiotic and class 3 truncated hemoglobin-like genes are differentially expressed in stone fruit rootstocks (Prunus L.) with different degrees of tolerance to root hipoxia. Tree Genetics \& Genome 9:1051-1063.

Appleby, C.A. 1992. The origin and functions of haemoglobin in plants. Science Progress 76:365-398.

Appleby, C.A., J.D. Tjepkema, and M.J. Trinick. 1983. Hemoglobin in a nonleguminous plant, Parasponia: possible genetic origin and function in nitrogen fixation. Science 220:951-953.

Arredondo-Peter, R., M.S. Hargrove, J.F. Moran, G. Sarath, and R.V. Klucas. 1998. Plant hemoglobins. Plant Physiology 118:1121-1126.

Arredondo-Peter, R., M.S. Hargrove, G. Sarath, J.F. Moran, J. Lohrman, J.S. Olson, et al. 1997. Rice hemoglobins: gene cloning, analysis and oxygen-binding kinetics of a recombinant protein synthesized in Escherichia coli. Plant Physiology 115:1259-1266.

Bhattacharya, S., A. Sen, S. Thakur, and L.S. Tisa. 2013. Characterization of haemoglobin from Actinorhizal plants- An in silico approach. Journal Bioscience 38:777-787.

Blokhina, O., and K.V. Fagerstedt. 2010. Reactive oxygen species and nitric oxide in plant mitochondria: origin and redundant regulatory systems. Physiologia Plantarum 138:447-462.

Bogusz, D., C.A. Appleby, J. Landsmann, E.S. Dennis, M.J. Trinick, and W.J. Peacock. 1988. Functioning haemoglobin genes in nonnodulating plants. Nature 331:178-180.

Bogusz, D., J. Llewellyn, S. Craig, E.S. Dennis, C.A. Appleby, and W.J. Peacock. 1990. Nonlegume hemoglobin genes retain organspecific expression in heterologous transgenic plants. Plant Cell 2:633-641.

Botrel, A., and W.M. Kaiser. 1997. Nitrate reductase activation state in barley roots in relation to the energy and carbohydrate status. Planta 201:496-501.
Bruno, S., S. Faggiano, F. Spyrakis, A. Mozzarelli, S. Abbruzzetti, E. Grandi, et al. 2007. The reactivity with $\mathrm{CO}$ of AHb1 and AHb2 from Arabidopsis thaliana is controlled by the distal HisE7 and internal hydrophobic cavities. Journal of the American Chemical Society 129:2880-2889.

Burgin, A.J., and S.K. Hamilton. 2007. Have we overemphasized the role of denitrification in aquatic ecosystems? A review of nitrate removal pathways. Frontiers in Ecology and the Environment 5:89-96.

Bustos-Sanmamed, P., A. Tovar-Méndez, M. Crespi, S. Sato, S. Tabata, and M. Becana. 2011. Regulation of nonsymbiotic and truncated hemoglobin genes of Lotus japonicus in plant organs and in response to nitric oxide and hormones. New Phytologist 189:765-776.

Bykova, N.V., A.U. Igamberdiev, W. Ens, and R.D. Hill. 2006. Identification of an intermolecular disulfide bond in barley hemoglobin. Biochemical and Biophysical Research Communications 347:301-309.

Couture, M., H. Chamberland, B. St. Pierre, J. LaFontaine, and M. Guertin. 1994. Nuclear gene encoding chloroplast hemoglobins in the unicellular green alga Chlamydomonas eugametos. Molecular and General Genetics 243:185-197.

Crawford, R.M.M. 1978. Metabolic adaptations to anoxia. p. 119136. In Hook, D.D., and R.M.M. Crawford (eds.) Plant life in anaerobic environments. Ann Arbor Science Publishers, Ann Arbor, Michigan, USA

Dordas, C. 2009. Nonsymbiotic hemoglobins and stress tolerance in plants. Plant Science 176:433-440.

Dordas, C., B.B. Hasinoff, A.U. Igamberdiev, N. Manac'h, J. Rivoal, and R.D. Hill. 2003. Expression of a stress-induced hemoglobin affects NO levels produced by alfalfa root cultures under hypoxic stress. Plant Journal 35:763-770.

Dordas, C., B.B. Hasinoff, J. Rivoal, and R.D. Hill. 2004. Class-1 hemoglobins, nitrate and NO levels in anoxic maize cellsuspension cultures. Planta 219:66-72.

Doyle, M.P., and J.W. Hoekstra. 1981. Oxidation of nitrogen oxides by bound dioxygen in hemoproteins. Journal of Inorganic Biochemistry 14:351-358.

Duff, S.M.G., J.B. Wittenberg, and R.D. Hill. 1997. Expression, purification, and properties of recombinant barley (Hordeum sp.) hemoglobin: Optical spectra and reactions with gaseous ligands. Journal of Biological Chemistry 272:16746-16752.

Farrés, J., M.P. Rechsteiner, S. Herold, A.D. Frey, and P.T. Kallio. 2005. Ligand binding properties of bacterial hemoglobins and flavohemoglobins. Biochemistry 44:4125-4134.

Gardner, P.R., A.M. Gardner, L.A. Martin, and A.L. Salzman. 1998. Nitric oxide dioxygenase: an enzymic function for flavohemoglobin. Proceedings of the National Academy Science of United States of America 95:10378-10383.

Garrocho-Villegas, V., S.K. Gopalasubramaniam, and R. ArredondoPeter. 2007. Plant hemoglobins: what we know six decades after their discovery. Gene 398:78-85.

Gupta, K.J., K.H. Hebelstrup, L.A.J. Mur, and A.U. Igamberdiev. 2011. Plant hemoglobins: Important players at the crossroads between oxygen and nitric oxide. FEBS Letters 585:3843-3849.

Guy, P.A., J-P. Sidaner, S. Schroeder, M. Edney, A.W. MacGregor, and R.D. Hill. 2002. Embryo phytoglobin gene expression as a measure of germination in cereals. Journal of Cereal Science 36:147-156.

Hargrove, M., E.A. Brucker, B. Stec, G. Sarath, R. Arredondo-Peter, R.V. Klucas, et al. 2000. Crystal structure of a nonsymbiotic plant hemoglobin. Structure 8:1005-1014.

Hebelstrup, K.H., A.U. Igamberdiev, and R.D. Hill. 2007. Metabolic effects of hemoglobin gene expression in plants. Gene 398:86-93.

Hebelstrup, K.H., E. Østergaard-Jensen, and R.D. Hill. 2008. Bioimaging techniques for subcellular localization of plant hemoglobins and measurement of hemoglobin-dependent nitric oxide scavenging In Planta. Methods in Enzymology 437:595-604. 
Hebelstrup, K.H., P. Hunt, E. Dennis, S.B. Jensen, and E.Ø. Jensen. 2006. Hemoglobin is essential for normal growth of Arabidopsis organs. Physiologia Plantarum 127:157-166.

Hill, R.D. 1998. What are hemoglobins doing in plants? Canadian Journal of Botany 76:707-712.

Holmberg, N., G. Lilius, J.E. Bailey, and L. Bülow. 1997. Transgenic tobacco expressing Vitreoscilla hemoglobin exhibits enhanced growth and altered metabolite production. Nature Biotechnology 15:244-247.

Hoy, J.A., and M.S. Hargrove. 2008. The structure and function of plant hemoglobins. Plant Physiology Biochemistry 46:371-379.

Hunt, P.W., E.J. Klok, B. Trevaskis, R.A. Watts, M.H. Ellis, W.J. Peacock, et al. 2002. Increased level of hemoglobin 1 enhances survival of hypoxic stress and promotes early growth in Arabidopsis thaliana. Proceedings of the National Academy of Sciences of United States of America 99:17197-17202.

Hunt, P.W., R.A. Watts, B. Trevaskis, D.J. Llewelyn, J. Burnell, E.S. Dennis, et al. 2001. Expression and evolution of functionally distinct haemoglobin genes in plants. Plant Molecular Biology 47:677-692.

Igamberdiev, A.U., K. Baron, N. Manac'h-little, M. Stoimenova, and R.D. Hill. 2005. The Haemoglobin/nitric oxide cycle: Involvement in flooding stress and effects on hormone signalling. Annals of Botany 96:557-564.

Igamberdiev, A.U., N.V. Bykova, and R.D. Hill. 2011. Structural and functional properties of class 1 plant hemoglobins. IUBMB Life 63(3):146-152.

Igamberdiev, A.U., and R.D. Hill. 2004. Nitrate, NO and haemoglobin in plant adaptation to hypoxia: An alternative to classic fermentation pathways. Journal of Experimental Botany 55:2473-2482.

Ioanitescu, A.I., S. Dewilde, L. Kiger, M.C. Marden, L. Moens, and S. Van Doorslaer. 2005. Characterization of nonsymbiotic tomato hemoglobin. Biophysical Journal 89:2628-2639.

Jakob, W., D.A. Webster, and P.M. Kroneck. 1992. NADH-dependent methemoglobin reductase from the obligate aerobe Vitreoscilla: improved method of purification and reexamination of prosthetic groups. Archives of Biochemistry and Biophysics 292:29-33.

Jokipii-Lukkari, S., A.D. Frey, P.T. Kallio, and H. Häggman. 2009. Intrinsic non-symbiotic and truncated haemoglobins and heterologous Vitreoscilla haemoglobin expression in plants. Journal of Experimental Botany 60:409-422.

Kubo, H. 1939. Über hämoprotein aus den wurzelknöllchen von leguminosen. Acta Phytochimica 11:195-200.

Kumar, A., M. Nag, and S. Basak. 2013. Truncated or $2 / 2$ hemoglobins: a new class of globins with novel structure and function. Journal of Proteins and Proteomics 4:45-64.

Kundu, S., S.A. Premer, J.A. Hoy, J.T. Trent, and M.S. Hargrove. 2003. Direct measurement of equilibrium constants for highaffinity hemoglobins. Biophysical Journal 84:3931-3940.

Li, X., R.H. Peng, H.Q. Fan, A.S. Xiong, Q.H. Yao, Z.M Cheng, et al. 2005. Vitreoscilla hemoglobin overexpression increases submergence tolerance in cabbage. Plant Cell Reports 23:710-715.

Lira-Ruan, V., G. Sarath, R.V. Klucas, and R. Arredondo-Peter. 2001. Synthesis of hemoglobins in rice (Oryza sativa var. Jackson) plants growing in normal and stress conditions. Plant Science 161:279-287.

Manac'h-Little, N., A.U. Igamberdiev, and R.D. Hill. 2005 Hemoglobin expression affects ethylene production in maize cell cultures. Plant Physiology and Biochemistry 43:485-489.

Mattana, M., C. Vannini, L. Espen, M. Bracale, A. Genga, M. Marsoni, et al. 2007. The rice Mybleu transcription factor increases tolerance to oxygen deprivation in Arabidopsis plants. Physiologia Plantarum 131:106-121.

Nie, X.Z., and R.D. Hill. 1997. Mitochondrial respiration and hemoglobin gene expression in barley aleurone tissue. Plant Physiology 114:835-840.
Ohwaki, Y., M. Kawagishi-Kobayashi, K. Wakasa, S. Fujihara, and T. Yoneyama. 2005. Induction of class-1 non-symbiotic hemoglobin genes by nitrate, nitrite and nitric oxide in cultured rice cells. Plant and Cell Physiology 46:324-331.

Ott, T., J.T. van Dongen, C. Gúnther, L. Krusell, G. Desbrosses, H Vigeolas, et al. 2005. Symbiotic leghemoglobins are crucial for nitrogen fixation in legume root nodules but not for general plant growth and development. Current Biology 15:531-535.

Perazzolli, M., P. Dominici, M.C. Romero-Puertas, E. Zago, A. Zeier, M. Sonoda, et al. 2004. Arabidopsis nonsymbiotic hemoglobin AHb1 modulates nitric oxide bioactivity. Plant Cell 16:2785-2794

Poole, R.K., and M.N. Hughes. 2000. New functions for the ancient globin family: bacterial responses to nitric oxide and nitrosative stress. Molecular Microbiology 36:775-783.

Qu, Z-L., N-Q. Zhong, H-Y. Wang, A-P. Chen, G-L. Jian, and G-X. Xia. 2006. Ectopic expression of the cotton non-symbiotic hemoglobin gene GhHbd1 triggers defense responses and increases disease tolerance in Arabidopsis. Plant and Cell Physiology 47:1058-1068.

Ross, E.J.H., J.M. Stone, C.G. Elowsky, R. Arredondo-Peter, R.V Klucas, and G. Sarath. 2004. Activation of the Oryza sativa nonsymbiotic haemoglobin-2 promoter by the cytokinin-regulated transcription factor, ARR1. Journal of Experimental Botany 55:1721-1731.

Saenz-Rivera, J., G. Sarath, and R. Arredondo-Peter. 2004 Modeling the tertiary structure of a maize (Zea mays ssp. mays) non-symbiotic hemoglobin. Plant Physiology and Biochemistry 42:891-897.

Sairam, R.K., V. Chinnusamy, A. Arora, P. Bhattacharya, R. Joshi, and S. Trivedi. 2012. Non-symbiotic hemoglobin and nitrate reductase constitute an alternative to fermentation in waterlogging tolerance of mung vean (Vigna radiata (L.) Wilczek). Indian Journal Plant Physiology 17:93-102.

Sairam, R.K., D. Kumutha, and K. Ezhilmathi. 2009. Waterlogging tolerance: nonsymbiotic haemoglobin-nitric oxide homeostasis and antioxidants. Current Science 96:674-682.

Sakamoto, A., S.H. Sakurao, K. Fukunaga, T. Matsubara, M Ueda-Hashimoto, S. Tsukamoto, et al. 2004. Three distinct Arabidopsis hemoglobins exhibit peroxidase-like activity and differentially mediate nitrite-dependent protein nitration. FEBS Letters 572:27-32.

Sasaki, T., J. Song, Y. Koga-Ban, E. Matsui, F, Fang, H. Higo, et al. 1994. Toward cataloguing all rice genes: large scale sequencing of randomly chosen rice cDNAs from a callus cDNA library. Plant Journal 6:615-624.

Sasakura, F., T. Uchiumi, Y. Shimoda, A. Suzuki, K. Takenouchi, S Higashi, et al. 2006. A class 1 hemoglobin gene from Alnus firma functions in symbiotic and nonsymbiotic tissues to detoxify nitric oxide. Molecular Plant-Microbe Interactions 19:441-450.

Serégelyes, C., L. Mustárdy, F. Ayaydin, L. Sass, L. Kovács, G Endre, et al. 2000. Nuclear localization of a hypoxia inducible novel non-symbiotic hemoglobin in cultured alfalfa cells. FEBS Letters 482:125-130.

Smagghe, B.J., S. Kundu, J.A. Hoy, P. Halder, T.R. Weiland, A. Savage, et al. 2006. Role of phenylalanine B10 in plant nonsymbiotic hemoglobins. Biochemistry 45:9735-9745.

Smagghe, B.J., J.T. Trent, and M.S. Hargrove 2008. NO dioxygenase activity in hemoglobins is ubiquitous in vitro, but limited by reduction in vivo. PLoS ONE 3:e2039.

Sowa, A., S.M.G. Duff, P.A. Guy, and R.D. Hill. 1998. Altering hemoglobin levels changes energy status in maize cells under hypoxia. Proceedings of the National Academy of Sciences of United States of America 95:10317-10321.

Spyrakis, F., S. Bruno, A. Bidon-Chanal, F.J. Luque, S. Abbruzzetti, C. Viappiani, et al. 2011b. Oxygen binding to Arabidopsis thaliana $\mathrm{AHb} 2$ nonsymbiotic hemoglobin: Evidence for a role in oxygen transport. IUBMB Life 63:355-362. 
Spyrakis, F., J. Luque, and C. Viappiani. 2011a. Structural analysis in nonsymbiotic hemoglobins: What can we learn from inner cavities? Plant Science 181:8-13.

Stöhr, C., F. Strube, G. Marx, W.R. Ullrich, and P. Rockel. 2001. A plasma membrane-bound enzyme of tobacco roots catalyses the formation of nitric oxide from nitrite. Planta 212:835-841.

Taylor, E.R., X.Z. Nie, A.W. MacGregor, and R.D. Hill. 1994. A cereal haemoglobin gene is expressed in seed and root tissues under anaerobic conditions. Plant Molecular Biology 24:853-862.

Thiel, J., H. Rolletschek, S. Friedel, J.E. Lunn, T.H. Nguyen, R. Feil, et al. 2011. Seed-specific elevation of non-symbiotic hemoglobin AtHb1: beneficial effects and underlying molecular networks in Arabidopsis thaliana. BMC Plant Biology 11:48.

Thompson, J.D., T.J. Gibson, F. Plewniak, F. Jeanmougin, and D.G. Higgins. 1997. The clustal X windows interface: flexible strategies for multiple sequence alignment aided by quality analysis tools. Nucleic Acids Research 24:4876-4882.

Tiedje, J.M., A.J. Sexstone, D.D. Myrold, and J.A. Robinson. 1982. Denitrification: ecological niches, competition, and survival. Antonie van Leeuwenhoek Journal of Microbiology 48:569-583.

Trevaskis, B., R.A. Watts, C. Andersson, D. Llewellyn, M.S. Hargrove, J.S. Olson, et al. 1997. Two hemoglobin genes in Arabidopsis thaliana: The evolutionary origins of leghemoglobins. Proceedings of the National Academy of Sciences of United States of America 94:12230-12234

Wang, R., K. Guegler, S.T. LaBrie, and N.M. Crawford. 2000. Genomic analysis of a nutrient response in Arabidopsis reveals diverse expression patterns and novel metabolic and potential regulatory genes induced by nitrate. Plant Cell 12:1491-1510.

Wang, Y-H., L.V. Kochian, J.J. Doyle, and D.F. Garvin. 2003. Two tomato non-symbiotic haemoglobin genes are differentially expressed in response to diverse changes in mineral nutrient status. Plant Cell and Environment 26:673-680.
Watts, R.A., P.W. Hunt, A.N. Hvitved, M.S. Hargrove, W.J. Peacock, and E.S. Dennis. 2001. A hemoglobin from plants homologous to truncated hemoglobins of microorganisms. Proceedings of the National Academy of Sciences of United States of America 98:10119-10124.

Wittenberg, J.B., M. Bolognesi, B.A. Wittenberg, and M. Guertin. 2002. Truncated hemoglobins: a new family of hemoglobins widely distributed in bacteria, unicellular eukaryotes, and plants. Journal Biological Chemistry 277:871-874.

Wittenberg, J.B., and B.A Wittenberg. 1990. Mechanisms of cytoplasmic hemoglobin and myoglobin function. Annual Review of Biophysics and Biophysical Chemistry 19:217-241.

Yamasaki, H., and Y. Sakihama. 2000. Simultaneous production of nitric oxide and peroxynitrite by plant nitrate reductase: in vitro evidence for the NR-dependent formation of active nitrogen species. FEBS Letters 468:89-92.

Zhao, L., R. Gu, P. Gao, and G. Wang. 2008. A non-symbiotic hemoglobin gene from maize, $\mathrm{ZmHb}$, is involved in response to submergence, high-salt and osmotic stresses. Plant Cell, Tissue and Organ Culture 95:227-237.

Zhu, H., and A.F. Riggs. 1992. Yeast flavohemoglobin is an ancient protein related to globins and a reductase family Fnr, a global transcriptional regulator of Escherichia coli, activates the Vitreoscilla hemoglobin ( $\mathrm{VHb}$ ) promoter and intracellular $\mathrm{VHb}$ expression increases cytochrome d promoter activity. Proceedings of the National Academy of Sciences of United States of America 89:5015-5019. 